\section{Root system performance}

Plant Root Sytems: Their Function and Interaction with the Soil. By R. Scott Russell. Pp. 298. (McGraw-Hill : London, 1977.) $£ 10$.

THE past few years have seen the appearance of three large volumes dealing with plant roots, in particular with their structure, function and interaction with the soil. The appearance of these books at the same time is no coincidence: it reflects a growing awareness of the very important, though often indirect, effects root system performance can have on plant production. At the present time there is a place for a book which reviews much of the current knowledge represented (for example, by these large symposia volumes) and at the same time putting the results in a practical context. $R$. Scott-Russell's new book fulfils this role admirably.

It is appropriate that Scott-Russell should be the person to write such a book. As Director of the Letcombe Laboratory, he has guided much of the exciting and revealing work on roots and root systems which has been carried out in the past two decades. Many of the studies at the Letcombe Laboratory have attempted to link observations of roots and root system. behaviour in manipulative laboratory experiments, with field observations; the ultimate aim of this work has been to highlight conditions which can limit root and subsequently crop growth.

Scott-Russell adopts a similar approach in his book, which has consequently been divided quite naturally into three parts. The first part describes root systems and their functions when no particular limit to root growth exists. This section, therefore, draws together much that is known about root system sizes in relation to aboveground parts, the growth and form that root systems take, the uptake of water and nutrients, and finally the relationships between roots and the rhizosphere flora.

The second part deals with the interaction between plant roots and soil. This section is introduced by a succinct chapter on the soil environment. Some might say too succinct but I feel that throughout the book the author has well realised where a fuller treatment would only serve to duplicate well known texts. Other chapters follow, covering some of the limiting conditions imposed in soils; chapters are therefore devoted to the effects of mechanical impedance on root growth and to the effects of anaerobic soils. The final chapter in this section deals with the soil/root interface and is particularly useful as it brings together much of the work in this somewhat 'new' research area.

The final section of the book deals with soil tillage and particularly directs attention at the possibility and consequence of reducing the level of cultivation soils receive. This is a topical subject, as there is currently a considerable increase in areas of land receiving lower levels of cultivation (despite the contrary suggestion in Fig. $12: 13$ of the book). These chapters draw on earlier parts of the book and illustrate quite clearly how an understanding and use of agricultural practices can be assisted by a thorough knowledge of plant and soil processes, with which they interact.

I thoroughly recommend this book to anyone seeking to understand

\section{Lymphocyte structure and function}

The Lymphocyte. Parts 1 and 2. Edited by J. J. Marchalonis. Pp. 1-369 and 373-704 (Marcel Dekker: Basel and New York, 1977.) SFr. 123 and 130.

IF definition is required, The Lymphocyte falls somewhere between a collection of reviews and a textbook; as such, is already obsolescent, as many of will perfectly fulfil neither role. As a collection of reviews, The Lymphocyte is already obsolescent, as many of the chapters were written four years ago. Judged as a textbook, it seems badly arranged. It has been chopped into somewhat arbitrary sections. Section three, for example, on "Physical Separation of Lymphocytes", contains but one short chapter of the same name. In the second volume, there is considerable overlap of material. Chapter 13, on "Ultrastructure of the Lymphocyte Surface", could quite readily have been omitted, as most of the material therein is given in other parts of the book. The text does, however, provide the student and those on the periphery of immunology with a wide range of information.

The ultrastructure of mammalian lymphocytes, and recirculating lymphocytes, are the two subjects of the first section of the book by T. E. Mandel and J. Sprent, respectively. Origins of lymphocytes and the lymphatic system are dealt with in the two chapters of the second section, one on the ontogenic emergence of immunocytes and the second on the phylogenetic emergence of lymphoid tissue and cells by $\mathrm{N}$. Cohen. In view of his statement that "Crocodilians have big teeth and have been neglected by immunologists", Dr Cohen evidently feels that a dash of current ideas on how plants function below ground. The text draws heavily from agricultural crop examples, perhaps because of the particular leanings of the author but more probably because there is much less data available on other plant types, for example, forest trees. Undergraduates in the plant and agricultural sciences would derive much benefit from this book, although its price might prove a deterrent. There are a few mistakes in the text but these are unimportant and unintrusive. The book is extremely well laid out with large clear print, and the use of different print types and print shades make reading very easy.

John M. Roberts

John M. Roberts is a Plant Physiologist working at the Institute of Hydrology, Wallingford, UK. humour in no way destroys good scientific literature.

The physical separation of lymphocytes by R. G. Miller is the short but nonetheless worthy topic of section three. The immune functions of lymphocytes are considered in section four which best achieves the object of the text since it gives a cross-section of current interests in the field. This section contains four chapters on such diverse topics as the influence of $\mathrm{T}$ cells on antibody formation and tolerance, cellular immune reactions, cell interactions in the in vitro immune response, and immune response genes.

The second volume of the book deals entirely with the lymphocyte plasma membrane, reflecting the major interest of the editor. The first chapter by Marchalonis himself deals with the purification of lymphocyte plasma membranes and the properties of lymphocyte surface immunoglobulins. This latter subject is also dealt with in two other chapters, one on lymphocyte surface antigens and the other on dynamic aspects of lymphocyte membranes. Other topics considered in this section include the lymphocyte coat or glycocalyx, lymphocyte membrane phospholipids, and mitogen stimulation of lymphocytes.

Thus, in summary, a wholehearted recommendation could not be given to this book partly because it is so dated and partly because better editing could have reduced its duplications. The publisher has mainly done a good job, although the quality of the figures is poor. The price, as so often happens these days, puts it into the grasp of the librarian and the reviewer rather than the private owner.

T. D. Heath

T. D. Heath is a research fellow in the Division of Biology of the Institute of Cancer Research, working at the Chester Beatty Research Institute, London, UK. 